\title{
Performance and Energy Benchmarking of Spectral Solvers on Hybrid Multicore Machines
}

\author{
Tania Banerjee, Jacob Rabb and Sanjay Ranka \\ Department of Computer and Information Science and Engineering, \\ University of Florida, Gainesville, FL 32611 \\ \{tmishra, jrabb, ranka\}@ cise.ufl.edu
}

\begin{abstract}
Hybrid multicore processors (HMPs) are poised to dominate the landscape of the next generation of computing on the desktop as well as on exascale systems. HMPs consist of general purpose GPU cores along with specialized cores and are expected to provide benefits to a wide spectrum of applications at significantly lower energy requirements per FLOP (FLoatingpoint Operations per Second).

In this paper, we describe a comprehensive strategy for efficiently implementing a key kernel of spectral solvers on GPUs and HMPs consisting of CPU and GPUs. Our implementations represent significant computational improvements of the kernel on GPU. We also provide load balancing strategies for a combination of CPU and GPU cores. We show that depending upon whether performance or energy optimization is critical, separate load balancing strategies should be used on HMPs. We provide performance, power and energy evaluation as well as modeling and validation for a variety of CPU-GPU core combinations.
\end{abstract}

Keywords: Spectral Element Methods, Power and Energy Evaluation, Performance Benchmarking, Exascale, Hybrid Computing.

\section{INTRODUCTION}

High performance computing is becoming increasing constrained by the power and energy requirements. DOE has specified a maximum power of $20 \mathrm{MW}$ for exascale systems [2]. To put this power number into context, consider the world's fastest petascale supercomputer Tianhe-2, that consumes $17.8 \mathrm{MW}$ at its peak performance of 54.9 petaFLOPS. So to operate at exascale speed, the performance of a supercomputer like Tianhe- 2 has to increase 20 times with only nominal increase in power consumption.

Hybrid multicore processors (HMPs) have the potential to address these power and energy constraints. HMPs consist of a multicore processor with one or more coprocessor. The coprocessor may be a large number of Intel Xeon Phi cores or general purpose GPU cores, for example. The coprocessors are expected to provide benefits for a wide spectrum of applications at significantly lower energy requirements per FLOP.

Our goal in this paper is to obtain high performance implementations of spectral element methods (SEM) which involve matrix multiplications, on hybrid CPU-GPU architectures. Achieving high performance matrix multiplications for small matrices is significantly more challenging than larger matrices as the computation to data access requirements are significantly lower than those for larger matrices. Another important goal of this paper is to study the power and energy requirements of these methods. SEM methods, relying on dense matrix multiplication, are expected to form the core computation of a number of applications targeted for exascale computing. CMT-nek is one such application of Computational Fluid Dynamics principles. Being based on Nek5000, a publicly available software using SEMs, CMT-nek reuses the computation kernels of Nek5000. CMT-nek has lighter communication patterns compared to Nek5000, hence the computation kernels could well be on the critical path to completion of the program. Therefore, we focus on performance and energy optimization strategies of the computation kernels, specifically on hybrid CPU-GPU compute nodes which are building blocks of the next generation of supercomputers.

The main contributions of this paper are as follows:

1) We develop improved implementations of the derivative computation kernels on GPUs that leverage the property of SEMs that for partial derivative computation in differential equations one of the two matrices in the matrix multiplications is identical for nearly all matrix multiplications. Our implementations are able to achieve 180 gigaFLOPS on a Tesla K20c architecture.

2) We develop empirical models of performance, power and energy consumption of the kernel on multiCPU and multiGPU systems. Although the theoretical complexity of the operations is $O\left(N^{4}\right)$, where the matrix size is $N \times N \times N$, our models show the true empirical complexity for performance, as well as for power and energy.

3) We derive load balancing techniques for dividing up the work on heterogeneous cores on a HMP, based on the empirical models of CPU and GPU cores. These techniques may be used to obtain either performance optimal or energy optimal implementation, depending upon the optimization objectives. We find that the computational workload assignment, in general, depends on whether the objective is to optimize for performance or for energy.

Throughout the paper we refer to the CPU core that hosts the GPU as the host and the GPU accelerator as the device.

The rest of the paper is described as follows. In Section II, we briefly describe the spectral methods in the context of Nek5000. Section III provides the optimization methods for the underlying small matrix multiplications on traditional GPU cores. Section IV provides experimental evaluation on a GPU and a combination of CPU+GPU architectures. In particular, Section IV-D3 presents load balancing strategy based on performance models and compares them to actual results, while Section IV-E4 gives the load balancing strategy for minimum overall energy consumption. Section V presents related work and we conclude in Section VI. 


\section{NEK5000}

NEK5000 [16] is a computational fluid dynamics solver for incompressible flows that is based on SEM. SEM is a highorder weighted residual technique that provides the geometric flexibility of finite elements, rapid convergence properties and tensor-product efficiency benefits of global structure methods [16]. NEK5000 code has been shown to scale to over a million CPU cores and was recognized with the Gordon Bell prize in 1999 for algorithmic quality and sustained parallel performance. Multiple elements are coupled through a structured or unstructured framework and inter-element coupling is achieved through matching of nodal interface values. The functions in a spectral element are represented as tensor products of $N$ th order Lagrangian bases on $n$ Gauss-Lobatto or Gauss quadrature points. The convergence for smooth functions is exponentially fast with local approximation of order $N$. The approach is also well suitable for a variety of compressible and incompressible flows due to minimal numerical dissipation and dispersion.

The tensor-product structure of SEM allows matrix-vector products to be computed as a sequence of matrix-matrix products. Efficient software for Matrix Multiplication is widely available for a variety of architectures. Given the good data locality characteristics of matrix multiplication, high performance can be achieved. Most of these implementations are targeted towards large matrices.

CMT-nek which is a (Compressible Multiphase Turbulence) simulation software is being developed at the PSAAP II center of the University of Florida. CMT-nek reuses the key SEM kernels of Nek5000. The computational domain is partitioned into hexahedral elements, where each three dimensional element is discretized using $N^{3}$ Gauss-Lobatto grid points. SEM discretization method is highly accurate and low in cost.

The spectral solver of CMT-nek code performs matrixvector multiplication to numerically compute partial derivatives of a function $U$, which is discretized as $u_{i j k}$ in three dimensional space. Each spectral element defines its own $U$. The derivatives of $U$ along the Cartesian coordinates $(r, s, t)$ are given by the following equations,

$$
\begin{aligned}
& \left.\frac{\partial U}{\partial r}\right|_{i, j, k}=\sum_{l=1}^{N_{x}} A_{i l} u_{l j k} \\
& \left.\frac{\partial U}{\partial s}\right|_{i, j, k}=\sum_{l=1}^{N_{y}} B_{j l} u_{i l k} \\
& \left.\frac{\partial U}{\partial t}\right|_{i, j, k}=\sum_{l=1}^{N_{z}} C_{k l} u_{i j l}
\end{aligned}
$$

where an element in three dimensional space is assumed to have $N_{x} \times N_{y} \times N_{z}$ Gauss-Lobatto grid points with values of $U$ being defined at those points. $A, B, C$ are the derivative operators, of dimensions $N_{x} \times N_{x}, N_{y} \times N_{y}$ and $N_{z} \times N_{z}$ respectively. If $N_{x}=N_{y}=N_{z}=N$ then we have $N^{3}$ grid points per element, and $B=C=A^{T} . B$ and $C$ are later represented as derivative operator matrix $D$, and $A$ is represented as $D^{T}$ in this paper. From the equations 1, 2 and 3 we observe that for computing these derivatives, the fastest changing index in $U$ are the first, middle and last indices respectively. If we analyze the computations, we will find that the memory access pattern for matrix $U$ is different for the three derivatives. For example, for computing partial derivatives along $r$, contiguous memory addresses are accessed, whereas for computing those along $s$ and $t$, memory is accessed in strides of $N_{x}$ and $N_{x} \times N_{y}$ addresses respectively. $N_{x}, N_{y}, N_{z}$ represent order of the polynomials that are fitted and values between 5 and 25 yield highly accurate results. Thus when Thus to compute derivatives of a function for all the elements, SEM requires the solution of a large number of matrix multiplications of very small matrices and we address efficient solutions for this problem in this paper.

The basic set of equivalent algorithms for computing partial derivatives along $(r, s, t)$, based on equations 1,2 and 3 are given in Figure 1. We analyze each of these algorithms in terms of performance, power and energy consumption.

\section{OPTIMIZATION STRATEGY}

The Basic Linear Algebra Subroutines (BLAS) libraries are optimized for large matrix multiplications. The BLAS routines however have non-optimal performance on small matrix multiplications. We present GPU and CPU+GPU optimization strategies in Sections III-A and III-B respectively.

\section{A. GPU Optimization}

Spectral methods require computing matrix multiplication for small matrices which is inherently challenging for most GPU type architectures as they have deep memory hierarchies that are not efficient when the compute to data access requirements is small. Libraries for multiplying matrices on NVIDIA GPUs (the main GPU target considered in this paper) are available as part of the Compute Unified Device Architecture (CUDA) toolkit version 4.1 in the cuBLAS library [17]. The batched version of the implementation (cublasXgemmBatched, $\mathrm{X}=\mathrm{S} / \mathrm{D} / \mathrm{C} / \mathrm{Z}$ ) is fast. However, the achieved performance is still low as compared to the peak GPU speed, unless the matrix sizes are multiples of 16 [8]. Several improvements of the above library are presented by Jhurani et al. in [8]. Their implementation provide significant improvements in performance over the cuBLAS implementation distributed in the CUDA Toolkit 5.0. Since their focus is on square matrices of size 16 and less they are directly relevant to higher order finite element methods including spectral elements methods.

Our implementation of the spectral solvers are based on the "TGEMM_multi_uniform" kernel (henceforth referred to as CUGEMM) described in [8]. We first created three separate kernels: one for each of the matrix multiplication patterns, $\frac{\partial U}{\partial r}$, $\frac{\partial U}{\partial s}$, and $\frac{\partial U}{\partial t}$. The $U$ matrices are laid out as one dimensional arrays, with the first index as the fastest changing dimension, the second index as the second fastest, and the third index as the slowest. Thus, the $\frac{\partial U}{\partial r}$ computation can be achieved by simply multiplying the operator matrix $D$ by contiguous $N \times N$ chunks of $U$. The $\frac{\partial U}{\partial s}$ computation can similarly 


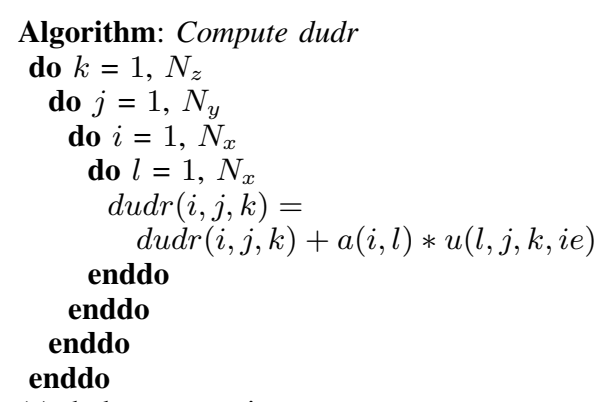

(a) dudr computation

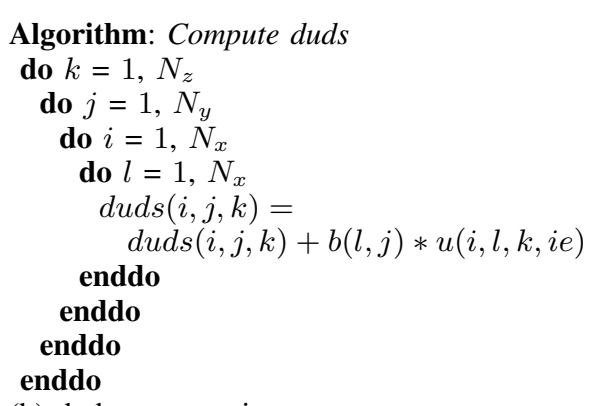

(b) duds computation

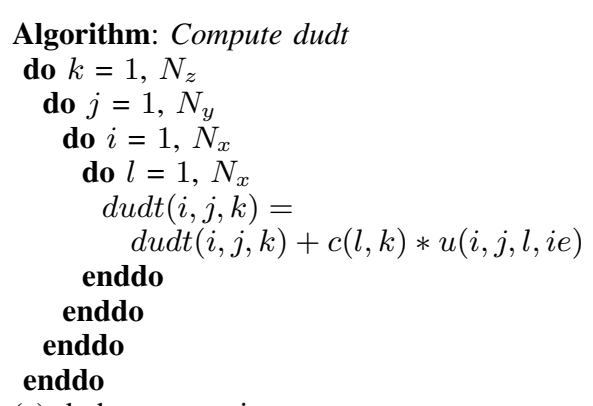

(c) dudt computation

Fig. 1. Algorithms to compute partial derivatives along directions $(r, s, t)$ for element with index $i e$.

be achieved by multiplying contiguous $N \times N$ chunks with the transpose of the $D$ matrix. However, for the $\frac{\partial U}{\partial t}$ case, there is no contiguous $N \times N$ chunk that would allow a matrix multiplication in the traditional sense, since the stride of accessing elements of $U$ is $N^{2}$. Instead, we have changed the access patterns of the $\frac{\partial U}{\partial t}$ kernel so that each SM computes elements along the $r$ and $t$ dimension. In other words, the threads step by one, and after $N$ threads, take a $N^{2}$ step.

There were two important optimization in our code to improve the overall efficiency for the target spectral solvers:

1) The derivative operator matrices $D$ and $D^{T}$ are only brought once per block from the device memory to shared memory. This reduces the number of memory transactions required.

2) The derivative operator matrices $D$ and $D^{T}$ are stored in registers instead of shared memory. This reduces the number of accesses to the shared memory.

Although our implementation is based on CUDA, the same optimization techniques can be applied in OpenCL[28] as well. Our experimental results show that the use of these two optimizations results in significantly better performance.

\section{B. $C P U+G P U$ Optimization}

The algorithm for derivative computation kernel on CPU is optimized using autotuning techniques described in [3]. As part of CPU+GPU optimization, we now describe our strategy for distributing workload among the processing units.

The derivative computation code of CMT-nek spectral solver is highly parallel in nature. This can be explained by the fact that the domain being modeled is partitioned into a large number of spectral elements, and derivative computation for each element is independent of the other elements. Further, the partial derivatives can be computed in parallel as well, for a given element. Also these partial derivatives are computed for a variety of functions and when these functions are independent, we can initiate derivative computation kernels for these functions in parallel for an element. Thus there is a tremendous potential of exploiting parallelism in this problem.

The highly parallel nature of the code simplifies workload partitioning. In our approach to partition workloads, the spectral elements are assigned to the processing units (CPU/GPU), which then compute the necessary partial derivatives for those elements. Assuming we have plenty of elements, this approach is more flexible in terms of distributing workloads to any number of processing units. If the number of spectral elements are few, then an alternate approach of distributing the computation of the partial derivatives to the processing units could be adopted. In this strategy, the smallest unit of task is a partial derivative computing kernel which could be $d u d r, d u d s$ or $d u d t$ for an element and these are assigned to the processing units to achieve load balancing. Since the matrices in spectral element method are typically small dense matrices, tiling them and assigning the tiles to the processing units is suboptimal in terms of workload partitioning.

In either approach, optimal assignment of tasks is determined offline. We use OpenMP in our work and distribute the elements using the \#pragma omp section directive. While the first thread invokes the GPU kernel, the other threads are assigned a certain number of tasks for processing the spectral elements. The optimal load balancing strategies on CPU and GPU for performance and energy are further explained in Section IV-D3 and Section IV-E4, respectively.

\section{EXPERIMENTAL RESUlts}

We performed our experiments using the derivative computation kernel of NEK5000 that involves double precision matrix-matrix multiply algorithms on a HMP. We consider $N=N_{x}=N_{y}=N_{z}$ in our experiments, and that values of $N$ lie between 5 and 16 which is sufficient for good accuracy of the numerical results. We first begin with a description of our platform in Section IV-A, followed by a statistical analysis of the collected data to give the reader an idea about the errors in measurement. This is followed by the GPU optimization results in Section IV-C. We then present performance, power and energy models on CPU, GPU and hybrid platforms in Section IV-D and the experimental results on our hybrid platform in Section IV-E.

\section{A. Platform}

Our experiments were run on an AMD Opteron 6168 host with $12 \mathrm{CPU}$ cores, running at $1.9 \mathrm{GHz}$ clock frequency. The processor is connected to a Tesla K20c GPU (Kepler architecture) via a PCI Express slot.

Tesla K20c has 13 multiprocessors, with 192 CUDA cores per multiprocessor. Core clock speed is $706 \mathrm{MHz}$, while memory clock speed is $2.6 \mathrm{GHz}$. The card has $48 \mathrm{k}$ shared memory per block, and $64 \mathrm{k}$ registers per block. This card has a peak performance of $1170 \mathrm{GFLOP} / \mathrm{s}$.

The optimization described in Section III-A exploit the memory access patterns for the function matrix $U$ and deriva- 
tive operator matrices $D$ and $D^{T}$. We modeled the base codes in $\mathrm{C}$ and compiled using nvcc in CUDA 5.0, V0.2.1221, with optimization flag "-O3". We compare our results with CUGEMM, Magma DGEMM and Cublas DGEMM. We tested two variants of the code computing derivatives. The first variant is referred to as 'Combined' and represents a case where the calculations are made faster by combining the three kernels for computing $\frac{\partial U}{\partial r}, \frac{\partial U}{\partial s}$ and $\frac{\partial U}{\partial t}$ into one kernel. This reduces the total number of memory transactions needed as $\frac{\partial U}{\partial r}$ and $\frac{\partial U}{\partial s}$ can use the same $N_{x} \times N_{y}$ sections of the $U$ matrix and the same operator matrix. However, this approach requires the computations for $\frac{\partial U}{\partial s}$ to conform to the memory access patterns for matrix $U$ in the computations for $\frac{\partial U}{\partial r}$. This causes the kernel to experience sub-optimal access patterns. The second variant is referred to as 'Separate'. In this variant the three kernels are executed separately. We measure power using the NVidia System Management Interface (nvidia-smi) command line utility. The "power-draw" field from NVSMI $\log$ gives us the power at any given instant. We take power measurements once per second while running our test program. We ran the kernel for one minute so that steady state power is reached and took the power measurements from nvidia-smi. These measurements are used to compute the average energy of the test program. We also compute performance per watt by first computing the FLOP per second count for the runs and then dividing it by the measured power.

Our host platform is not instrumented for power measurement. Hence we approximate the power on the host by running the host code on an AMD Fusion platform instrumented with PowerInsight[10], by scaling down the clock frequency on this platform to $1.9 \mathrm{GHz}$. The host code was run for about a minute to capture steady state power using PowerInsight.

\section{B. Statistics on measurements}

For different combinations of the input parameters, namely matrix size $N$ and number of elements $Y$, we collected 1000 samples of time and power measurements on CPU and about 100 samples on GPU and present the results in Figure 2 and 3 respectively. The third and fourth columns in these tables show the average runtime in seconds and standard deviation, while columns fifth and sixth show the mean of total power and the corresponding standard deviation. The low standard deviation values in both the cases indicates that the uncertainty in the measured execution times and power requirements is small and that mean values can be effectively used for overall modeling of power and runtime. Hence, graphs and models for performance, energy and power requirements based on architecture and problem parameters for the rest of this paper is based on mean times from multiple samples calculated for each set of parameter values.

\section{GPU Experiments}

a) Performance: As shown in Figure 4, the overall GPU performance increases (nearly linearly) with increase in matrix size and show that GPUs are more efficient with larger matrix sizes. Additionally, the separate version is better than

\begin{tabular}{|l|l|l|l|l|l|}
\hline \multirow{2}{*}{$N$} & \multirow{2}{*}{$Y$} & \multicolumn{2}{l|}{ Performance (seconds) } & \multicolumn{2}{l|}{ Total power(W) } \\
\cline { 3 - 6 } & $Y$ & Mean & Std. dev. & Mean & Std. dev. \\
\hline 8 & 2000 & 0.029 & 0.000 & 70.230 & 0.950 \\
8 & 4000 & 0.058 & 0.001 & 68.125 & 0.918 \\
8 & 6000 & 0.087 & 0.001 & 71.319 & 0.592 \\
8 & 8000 & 0.115 & 0.002 & 68.529 & 0.921 \\
8 & 10000 & 0.144 & 0.002 & 71.739 & 0.972 \\
\hline 10 & 2000 & 0.089 & 0.001 & 68.456 & 0.819 \\
10 & 4000 & 0.178 & 0.002 & 68.987 & 0.765 \\
10 & 6000 & 0.266 & 0.002 & 69.005 & 0.568 \\
10 & 8000 & 0.357 & 0.003 & 68.837 & 0.786 \\
10 & 10000 & 0.443 & 0.004 & 68.835 & 0.694 \\
\hline 12 & 2000 & 0.136 & 0.001 & 69.162 & 0.853 \\
12 & 4000 & 0.274 & 0.002 & 69.358 & 0.762 \\
12 & 6000 & 0.406 & 0.003 & 69.492 & 0.501 \\
12 & 8000 & 0.593 & 0.006 & 69.518 & 0.561 \\
12 & 10000 & 0.676 & 0.005 & 69.693 & 0.493 \\
\hline 14 & 2000 & 0.247 & 0.002 & 70.127 & 0.824 \\
14 & 4000 & 0.491 & 0.003 & 70.349 & 0.706 \\
14 & 6000 & 0.734 & 0.005 & 70.415 & 0.614 \\
14 & 8000 & 0.981 & 0.007 & 70.495 & 0.670 \\
14 & 10000 & 1.222 & 0.009 & 70.616 & 0.568 \\
\hline 16 & 2000 & 0.548 & 0.004 & 67.972 & 0.449 \\
16 & 4000 & 1.093 & 0.008 & 68.073 & 0.500 \\
16 & 6000 & 1.638 & 0.013 & 68.125 & 0.429 \\
16 & 8000 & 2.193 & 0.017 & 68.132 & 0.400 \\
16 & 10000 & 2.733 & 0.028 & 68.116 & 0.396 \\
\hline
\end{tabular}

Fig. 2. Statistics on performance and power measurement on CPU. 1000 samples were used for each combination of polynomial order $N$ and number of elements $Y$.

\begin{tabular}{|l|l|l|l|l|l|}
\hline & & \multicolumn{2}{|l|}{ Performance (seconds) } & \multicolumn{2}{l|}{ Total power $(\mathrm{W})$} \\
\cline { 3 - 6 }$N$ & $Y$ & Mean & Std. dev. & Mean & Std. dev. \\
\hline 8 & 2000 & 0.007 & 0.000 & 124.619 & 0.481 \\
8 & 4000 & 0.013 & 0.000 & 126.152 & 0.117 \\
8 & 6000 & 0.021 & 0.000 & 126.088 & 0.100 \\
8 & 8000 & 0.030 & 0.002 & 126.795 & 0.085 \\
8 & 10000 & 0.039 & 0.001 & 126.832 & 0.152 \\
\hline 10 & 2000 & 0.013 & 0.001 & 129.124 & 0.406 \\
10 & 4000 & 0.025 & 0.002 & 128.507 & 0.099 \\
10 & 6000 & 0.038 & 0.002 & 130.323 & 0.122 \\
10 & 8000 & 0.052 & 0.000 & 130.255 & 0.113 \\
10 & 10000 & 0.062 & 0.005 & 130.559 & 0.068 \\
\hline 12 & 2000 & 0.021 & 0.002 & 125.530 & 0.087 \\
12 & 4000 & 0.044 & 0.003 & 126.699 & 0.089 \\
12 & 6000 & 0.067 & 0.000 & 126.534 & 0.119 \\
12 & 8000 & 0.087 & 0.007 & 126.185 & 0.101 \\
12 & 10000 & 0.110 & 0.006 & 126.098 & 0.656 \\
\hline 14 & 2000 & 0.034 & 0.003 & 123.717 & 0.192 \\
14 & 4000 & 0.067 & 0.007 & 122.337 & 7.627 \\
14 & 6000 & 0.104 & 0.008 & 125.544 & 0.075 \\
14 & 8000 & 0.143 & 0.008 & 126.059 & 0.084 \\
14 & 10000 & 0.162 & 0.014 & 126.669 & 0.168 \\
\hline 16 & 2000 & 0.054 & 0.000 & 118.056 & 0.267 \\
16 & 4000 & 0.103 & 0.009 & 118.281 & 0.266 \\
16 & 6000 & 0.162 & 0.001 & 118.657 & 0.163 \\
16 & 8000 & 0.201 & 0.020 & 118.847 & 0.184 \\
16 & 10000 & 0.245 & 0.021 & 119.023 & 0.279 \\
\hline
\end{tabular}

Fig. 3. Statistics on performance and power measurement on GPU. 100 samples were used for each combination of polynomial order $N$ and number of elements $Y$.

combined version for larger matrix sizes. For $16 \times 16 \times 16$ matrices, we achieve a $39 \%$ improvement over CUGEMM. When compared with MAGMA and CUBLAS DGEMM, our algorithm is over $98 \%$ more efficient.

b) Power: The actual power consumed were relatively similar for each kernel, so the power results (in GFLOPS/W) are mostly dependent on the performance improvements we achieved. Figure 5 shows the performance per watt graphs for the different matrix sizes. We obtained results for all block sizes between 4 and 16 . 


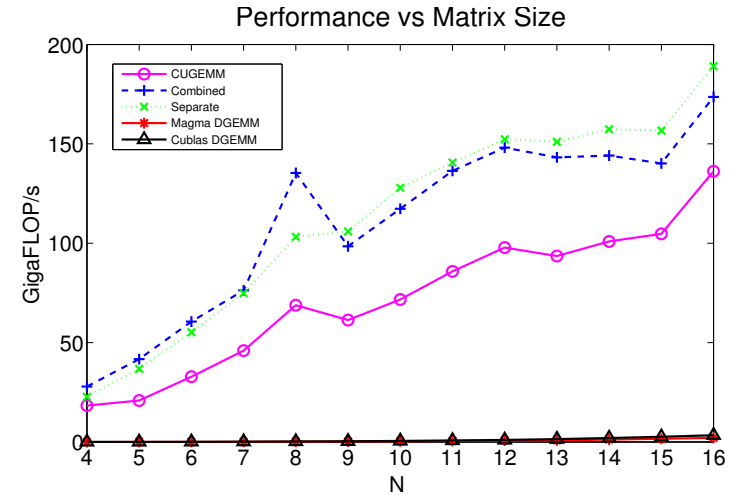

Fig. 4. Computation performance of different implementations of matrixmatrix multiply kernels on GPU in Giga FLOPs per second.

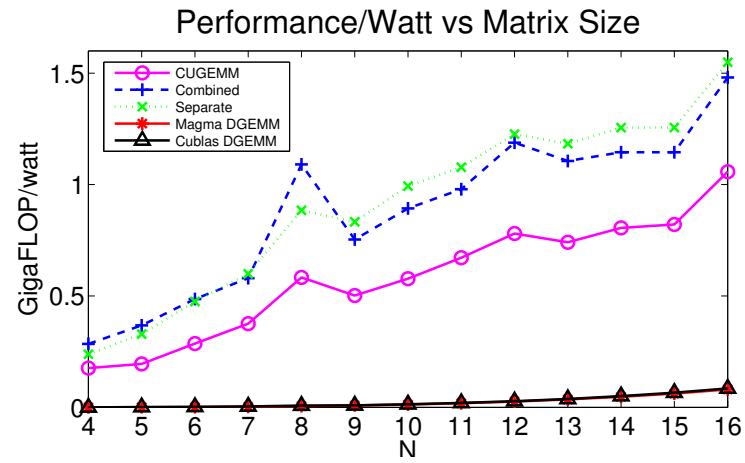

Fig. 5. Computation performance of different matrix-matrix multiply kernels on GPU in Giga FLOPs per Watt

c) Computation versus Communication: We studied the time taken to compute the matrix-matrix multiplications (computation time) and the time to transfer data between host and device (communication time). Communication involves transfer of the input matrices from the host to the device and the results from the device to the host. Communication happens over PCI Express (PCIe) which is a low latency, high bandwidth, packet-based switched point-to-point interconnect [22] and PCIe transfers between host and device consists typically of large messages sent over DMA. Figure

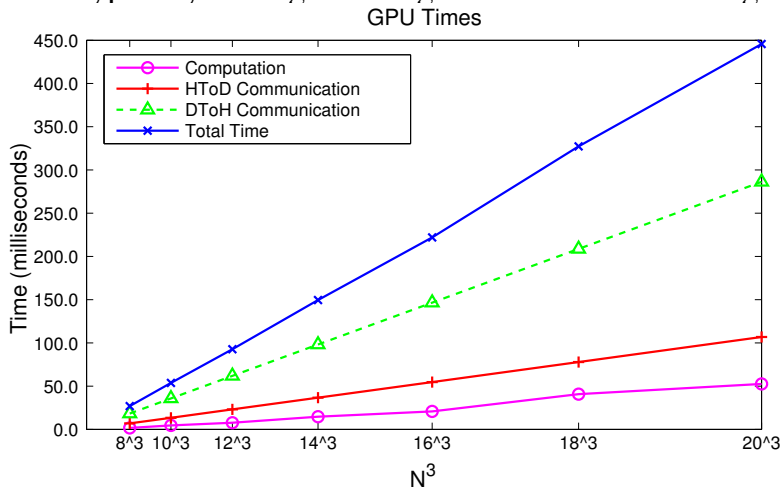

Fig. 6. Breakup of total GPU time into computation and communication components. Communication involves host to device and device to host data transfer over PCI Express. Communication time is proportional to the amount of data transferred. Computation time is a small fraction of the communication time.

shows the computation and communication times. From this graph we see that computation time is only a small fraction of the communication time. For example, for matrix size 8 , the ratio is $1 / 11$, for matrix size 16 its $1 / 7$ and for matrix size 20 its $1 / 5$. Hence, we don't overlap the data transfers with kernel execution in our implementation. Instead we use explicit memory copy statements [26] to first transfer the input matrix from host memory to device memory, then invoke GPU matrix multiplication kernel and finally copy the results back to the CPU.

\section{Modeling performance, power and energy consumption}

We model the GPU and CPU characteristics such as performance, power and energy consumption, based on two parameters: matrix size, $N$, and the number of elements $Y$. The modeling helps us gain insight into the relationship of performance characteristics of small matrix multiplications with respect to matrix size and the number of elements. The models helps us to understand the load balancing problem on CPU and GPU, and provides us with a good starting point to achieve optimum performance and energy consumption. The alternate method for load balancing using autotuning or binary search takes up significant machine time as the program has to be run several times to find the optimum load distribution to CPU and GPU. The models are also useful to theoretically analyze the characteristics of CPU-GPU configurations that are not available to us for direct experimentation. We present our GPU and CPU models in Sections IV-D1 and IV-D2, respectively. Performance on hybrid multi CPU-multi GPU systems is modeled and validated in subsection IV-D.

1) Modeling on GPU: The two components of GPU performance are computation time and communication time (Figure 6). Let $T_{\text {comp }}$ and $T_{\text {comm }}$ represent the computation and communication times respectively, and $T_{G P U}$ represent the total time taken by the derivative computation kernel to run on GPU. Using a linear least squares fit on a plot of logarithm of the data, its slope and the $y$-intercept gives us the exponent of the term $N$ (matrix size) and its coefficient respectively in our model. We similarly determine the exponent and coefficient of the term $Y$ (number of elements).
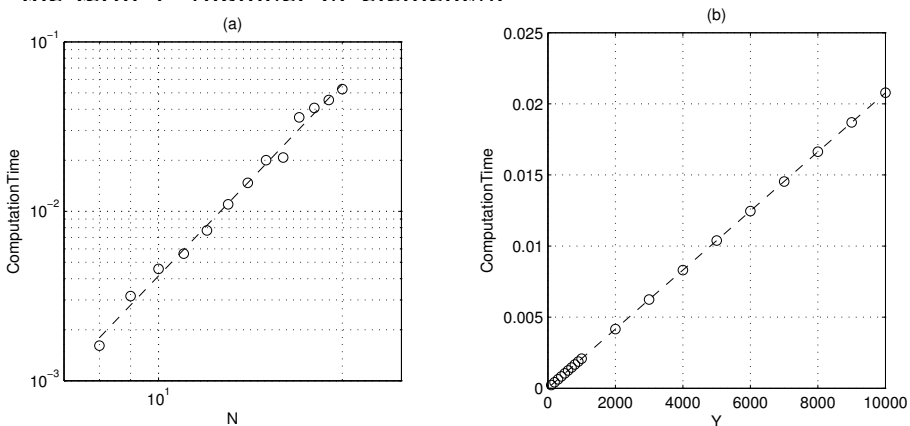

Fig. 7. (a) GPU computation time versus matrix size, $N$, on log-log plot, slope $=3.76$ (b) GPU computation time versus number of elements, $Y$, slope $=1$. For a timing model combining $N$ and $Y$ for GPU computation, $R^{2}=0.9974$, RMSE $=0.1311$ for the corresponding multiple regression model in Equation 4 .

Figures 7 and 8 show the actual plot as well as the least squares fit (in dashed line) for $T_{\text {comp }}$ and $T_{\text {comm }}$, respectively.

We noticed that while $T_{\text {comp }}$ is stable over repeated runs, $T_{\text {comm }}$ is not so. The value of $T_{\text {comm }}$ for each run depends 

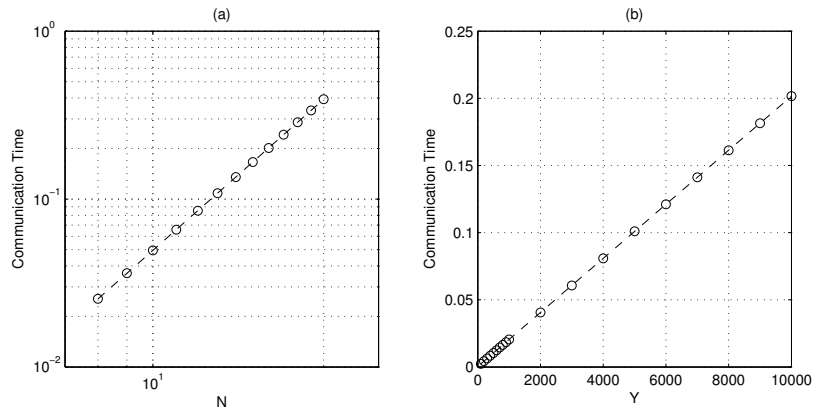

Fig. 8. (a) GPU communication time versus matrix size, $N$, slope=3 (b) GPU computation time versus number of elements, $Y$, slope $=1$. For timing model combining $N$ and $Y$ for GPU communication, $R^{2}=0.9934$, RMSE $=0.1935$, for the corresponding multiple regression model in Equation 5

on the location of the core running the host code. Since the CPU is based on non-uniform memory access (NUMA), the data packets may take an extra HyperTransport hop to reach the GPU depending upon which core is running the host code, introducing variability in communication time. To overcome this, we ran the host code on the same core by setting environment variable, GOMP_CPU_AFFINITY, appropriately.

Using multiple regression, we obtain combined models for $T_{\text {comp }}$ and $T_{\text {comm }}$ based on both $N$ and $Y$.

$$
\begin{aligned}
T_{c o m p} & =7.17 \times 10^{-11} \times N^{3.76} \times Y \\
T_{c o m m} & =6.14 \times 10^{-9} \times N^{3} \times Y \\
\text { Hence, } T_{G P U} & =T_{c o m p}+T_{c o m m} \\
& =7.17 \times 10^{-11} \times N^{3.76} \times Y \\
& +6.14 \times 10^{-9} \times N^{3} \times Y
\end{aligned}
$$

The coefficients of these equations are obtained from the $y$ intercept of the respective $\log$-log plot and represents the base level model value (power or timing) for unit matrix size and number of elements. These coefficients are dependent on system configuration.

To check how well the model fits the data, we analyzed Rsquared value and root-mean-square error (RMSE). R-squared is a statistical measure of how close the data are to the fitted regression line. Possible value of R-squared is between 0 and 1 , where 0 is the worst case in which no data is matched by the fitted line. For equation 4, R-squared is 0.9974 and RMSE is 0.1311 , and for equation 5, R-squared is 0.9934 and RMSE is 0.1935 .

From equation 4 we observe that $T_{\text {comp }}$ varies proportionally as $N^{3.76}$ which is close to the theoretical complexity of $O\left(N^{4}\right)$. It is worth noting that this empirical complexity is for small values of $N$ and is potentially due to differential access times for different sizes of $N$ because of memory hierarchy. As $N$ increases, we expect the behavior to be closer to $N^{4}$. However, for practical considerations of the code, the range of $N$ under consideration is expected to be smaller than 32 . The communication time, on the other hand, is proportional to the amount of data transferred, which in this case are the double precision numbers in three dimensional matrix of size $N \times N \times N$, making the complexity of communication time $O\left(N^{3}\right)$. Though computation has higher complexity than communication, the coefficient in the $T_{c o m p}$ equation 4 is two orders of magnitude smaller than that of communication time, $T_{\text {comm }}$, in equation 5 . This indicates that total runtime is dominated by the communication time between the host and the device at least for small matrix sizes. Both computation and communication times depend linearly on the number of spectral elements.

Using a similar strategy, we model GPU power and energy for the derivative computation kernel on our platform and these are given by the following equations obtained after performing multiple regression analysis.

$$
\begin{aligned}
& P_{G P U}=162.24 \times N^{-0.1} \\
& E_{G P U}=9.28 \times 10^{-7} \times N^{3} \times Y
\end{aligned}
$$

Energy should always be power times time. However, equation 8 is the best fit based on empirical data and closely follows the above requirement. For the power equation 7, R-squared is 0.5146 , though RMSE is 0.03929 . Power consumption does not depend on the number of elements processed, and overall power consumption decreases very slightly as larger matrices are used. This happens because of two factors namely, the number of memory transactions per unit of data transferred decreases and the computation to data transfer ratio improves with increasing $N$. For the energy equation 8, R-squared is 0.9941 , whereas RMSE is 0.182 . The energy consumption equation for $E_{G P U}$ is very similar to the equation for computing $T_{G P U}$, which shows that energy consumption is proportional to runtime.

When multiple GPUs are present, the workload could be distributed uniformly and for $g$ GPUs we approximate the performance trend to

$$
T_{g G P U}=1 / g \times T_{G P U}
$$

where $T_{G P U}$ is given by equation 6 . It is possible to achieve the $1 / g$ factor since the GPUs need not communicate with each other to solve this problem.

2) Modeling on CPU: CPU performance can be similarly modeled. The CPU implementation we used was optimized for each matrix size using autotuning[3].
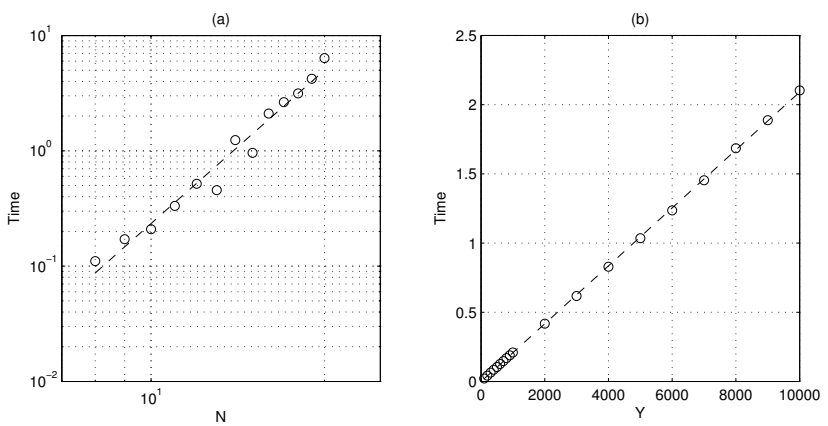

Fig. 9. (a) CPU time vs. $N$, slope $=4.4$ (b) $\mathrm{CPU}$ time vs. $Y$, slope $=1$.For a timing model combining $N$ and $Y$ for CPU computation, $R^{2}=0.9909$, $\mathrm{RMSE}=0.0719$, for the corresponding multiple regression model in Equation 10 . 
Figures 9(a) and (b) show the actual plot and the fitted lines for CPU time with respect to matrix size $N$ and the number of elements $Y$, respectively. Using multiple regression:

$$
T_{C P U}=1.02 \times 10^{-9} \times N^{4.4} \times Y
$$

Similarly, on our platform, CPU power and energy equations for the derivative computation kernel are given by the following equations.

$$
\begin{aligned}
P_{\text {memory }} & =5.95 \\
P_{\text {core }} & =12.21 \times N^{0.3} \\
P_{C P U} & =P_{\text {memory }}+P_{\text {core }} \\
E_{C P U} & =2.51 \times 10^{-8} \times N^{4.5} \times Y
\end{aligned}
$$

When multiple CPUs are present, the workload is distributed uniformly and runtime using $p$ CPUs is:

$$
T_{p C P U}=1 / p \times T_{C P U}
$$

3) Modeling on hybrid $(C P U+G P U)$ system: In this section we model performance of the derivative computation kernel on hybrid multiCPU, multiGPU systems based on the models we already developed in Sections IV-D1 and IV-D2. The model can be used for load balancing to determine optimal distribution of workload between the CPU cores and the GPUs and determine the minimum runtime of the combined system.

Since the work done by CPU core overlaps with that on the GPU, the total execution time is best modeled as

$$
T_{\text {eff }}=\max \left(T_{C P U}, T_{G P U}\right)
$$

Thus, $T_{\text {eff }}$ is minimized when $T_{C P U}=T_{G P U}$. This condition can be used to theoretically obtain the minimum runtime and also to determine the $Y$ 's in equations 6 and 10. For example, suppose $y_{c p u}$ and $y_{g p u}$ are the number of spectral elements processed by the CPU and the GPU respectively. Then if the total number of elements $\left(y_{c p u}+y_{g p u}\right)$ and the matrix size are given, one can easily solve for $y_{c p u}$ and $y_{g p u}$ from the condition $T_{C P U}=T_{G P U}$.

For a $p$-core CPU and $g$-GPU system,

$$
T_{e f f}=\max \left(T_{p C P U}, T_{g G P U}\right)
$$

to which we can similarly apply the minimization condition $T_{p C P U}=T_{g G P U}$ to get the minimum runtime as well as the distribution of workload among the processing units.

Figure 10 shows for different matrix sizes, $N \times N \times N$, and for different sets of $(p, g)$, the execution time (in seconds) on hybrid architectures using the empirical model and the optimum obtained using actual experiments for 10000 spectral elements, along with a percentage of the number of elements $y_{\text {срu }}$ processed by the CPU in each case. The last column in

\begin{tabular}{|c|c|c|c|c|c|c|}
\hline \multirow{2}{*}{$\begin{array}{l}\text { pCPU- } \\
\text { gGPU }\end{array}$} & \multirow{2}{*}{$\begin{array}{lr}\text { Matrix } & \text { size } \\
(N \times N \times N)\end{array}$} & \multicolumn{2}{|l|}{ Model } & \multicolumn{2}{|c|}{ Optimum } & \multirow{2}{*}{$\begin{array}{l}\text { Deviation } \\
\text { of } \\
\text { time }(\%)\end{array}$} \\
\hline & & $y_{\text {сри }} \%$ & time $(s)$ & $y_{\text {сри }} \%$ & time $(s)$ & \\
\hline \multirow{7}{*}{$1-1$} & $8 \times 8 \times 8$ & 24.02 & 0.0214 & 20.44 & 0.0218 & 1.83 \\
\hline & $9 \times 9 \times 9$ & 21.02 & 0.0314 & 18.44 & 0.0321 & 2.18 \\
\hline & $10 \times 10 \times 10$ & 18.58 & 0.0442 & 20.53 & 0.0430 & 2.79 \\
\hline & $11 \times 11 \times 11$ & 16.59 & 0.0601 & 17.25 & 0.059 & 1.86 \\
\hline & $12 \times 12 \times 12$ & 14.92 & 0.0794 & 15 & 0.0782 & 1.53 \\
\hline & $14 \times 14 \times 14$ & 12.32 & 0.1293 & 10.62 & 0.1349 & 4.15 \\
\hline & $16 \times 16 \times 16$ & 10.41 & 0.1968 & 9.75 & 0.2072 & 5.02 \\
\hline \multirow{7}{*}{$5-1$} & $8 \times 8 \times 8$ & 61.95 & 0.011 & 56.25 & 0.012 & 8.33 \\
\hline & $9 \times 9 \times 9$ & 57.77 & 0.017 & 53.12 & 0.0186 & 8.60 \\
\hline & $10 \times 10 \times 10$ & 53.97 & 0.0254 & 56.25 & 0.0239 & 6.28 \\
\hline & $11 \times 11 \times 11$ & 50.50 & 0.0363 & 51.56 & 0.0348 & 4.31 \\
\hline & $12 \times 12 \times 12$ & 47.34 & 0.0499 & 46.87 & 0.0497 & 0.40 \\
\hline & $14 \times 14 \times 14$ & 41.85 & 0.0869 & 37.49 & 0.0942 & 7.75 \\
\hline & $16 \times 16 \times 16$ & 37.27 & 0.1393 & 33.97 & 0.1524 & 8.60 \\
\hline
\end{tabular}
this table gives the deviation of the actual runtime from the predicted runtime.

Thus, the condition for minimizing runtime is that all the units must finish processing their load at about the same time. For minimizing energy consumption, on the other hand, we must minimize the sum of energy consumption of each individual unit. This is explained with an example from our
Fig. 10. Comparison of the runtime obtained for hybrid implementation of derivative computation for 10000 spectral elements with empirical model and optimum experimental time. $y_{\text {сри }} \%$ is the percentage of load on CPU. Model predicted time is within $10 \%$ of actual time.

experiments in Section IV-E4. One interesting observation there is that energy consumption of a hybrid system is not directly proportional to runtime. For the individual units though, energy consumption is directly proportional to runtime as we see from equations 8 and 14, where energy consumption $E_{G P U}$ and $E_{C P U}$ are proportional to runtimes $T_{G P U}$ and $T_{C P U}$ respectively.

\section{E. $C P U+G P U$ Experiments}

We now present the experimental results of a hybrid implementation of CMT-nek spectral element solver. Idle CPU cores are utilized in this implementation to compute the derivatives. Since there are $12 \mathrm{CPU}$ cores on our platform, we vary the number of CPU cores from 1 through 11 in our experiments, leaving aside one core to host the GPU. The experiments are based on strong scaling, where the total workload supplied to the system remains constant but the number of processing units is increased systematically. We studied performance, power and energy consumption characteristics of our implementation for a workload size of 10000 spectral elements.

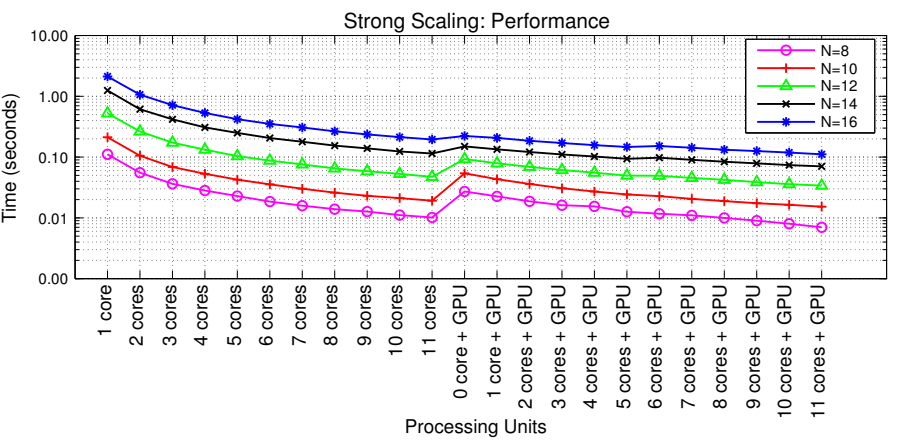

Fig. 11. Performance as 10000 spectral elements are processed by an increasing number of processing units, for different matrix sizes. Runtime decreases by $20 \%-90 \%$ as a GPU core is added to any given number of CPU cores

1) Performance Scaling: For matrix sizes $8 \times 8 \times 8,10 \times$ $10 \times 10,12 \times 12 \times 12,14 \times 14 \times 14$ and $16 \times 16 \times 16$, Figure 11 shows how processing time decreases as the more processing units are used. For CPU-only configurations runtime drops by 


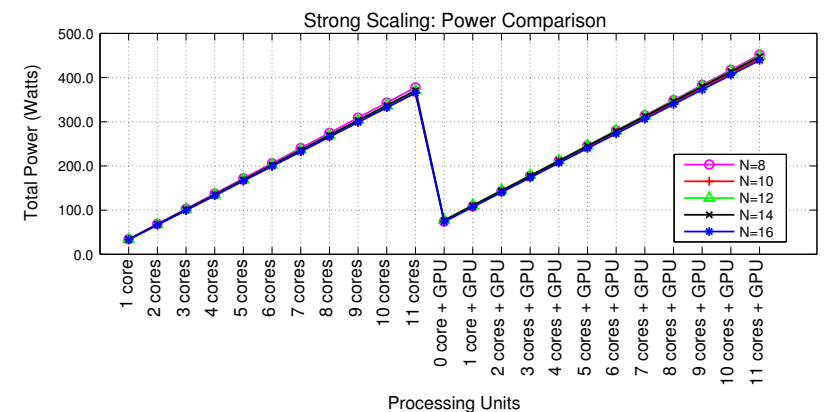

(a) Power consumed by the experiments in Figure 11. The range of power variation is from $63 \mathrm{~W}$ to $903 \mathrm{~W}$. Power consumption is not dependent on matrix size in any significant way and increases proportionally as more CPU cores are added.

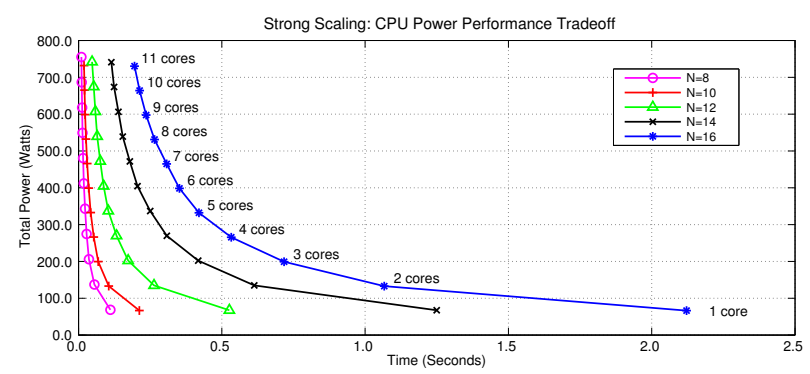

(b) Power performance tradeoff for the CPU-only configurations in Figure 11. Power consumed is the least when one core is used.

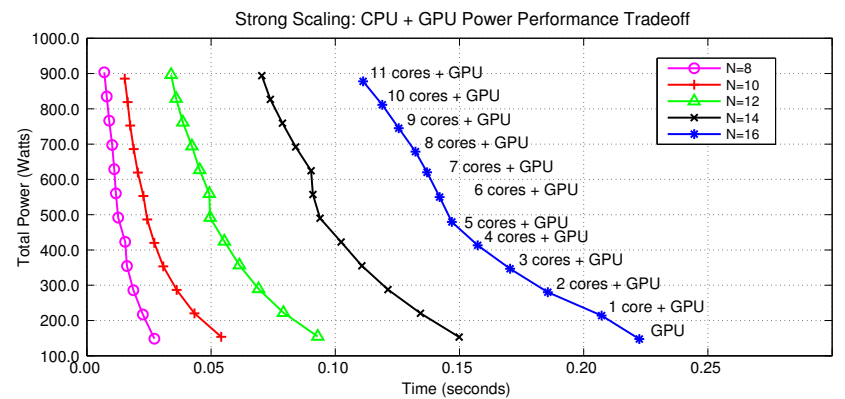

(c) Power performance tradeoff for the CPU+GPU configurations in Figure 11. Power consumed is least when only GPU is used.

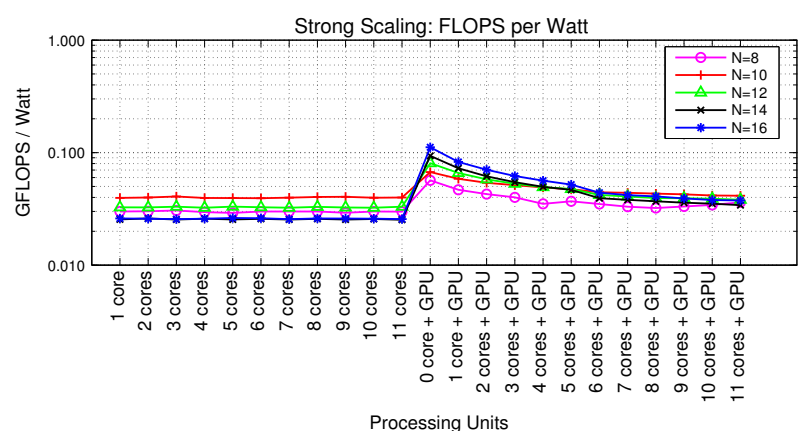

(d) Performance per Watt for different combinations of processing units and matrix sizes. CPU is more efficient for processing smaller matrices, whereas GPU is more efficient for processing larger matrices. As more CPU cores are added with the GPU, the efficiency of the overall system tend to get independent of matrix size.

Fig. 12. Power performance tradeoff under strong scaling

a factor of the number of cores used to process the load. For the CPU+GPU configurations, processing time decreases by

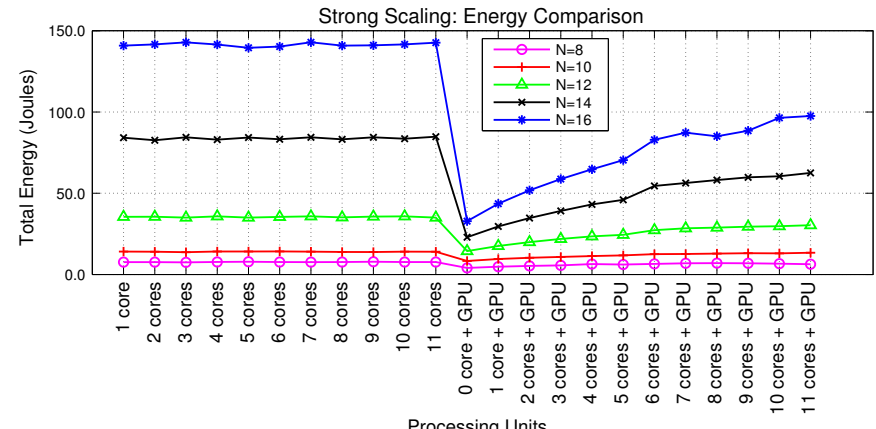

Fig. 13. Energy Consumption. Total energy remains unaffected in CPU-only configurations since increase in power is offset by a proportional decrease in runtime as more cores are used. With GPU, energy consumption increases as more $\mathrm{CPU}$ cores are used. Even then, energy consumption for 11 cores is about 1.5 times that for 11 cores + GPU.

20-90\% compared to the corresponding CPU only configurations.

2) Power: Figure 12(a) gives the power consumption for the different matrix sizes. While processing matrices of size $16 \times 16 \times 16$ consumes slightly lower power, power consumption generally does not depend upon the size of the matrices used in the multiplication routine. We assume here that the idle CPU cores can be shut off, and therefore, power consumption of idle CPU cores is $0 \mathrm{~W}$. We have made the assumption that cores can be completely shutoff for reasonable duration in performing this analysis. Clearly, when this is not the case a constant can be added to represent the idle power that will be used by all the cores. This value should generally not depend on the number of CPU cores that are used with or without the GPU and should, in general, offset the power requirement graphs or performance-energy tradeoff graphs by a constant amount.

Figures 12(b) and 12(c) gives the power-performance tradeoff for CPU-only and CPU+GPU configurations. Performance

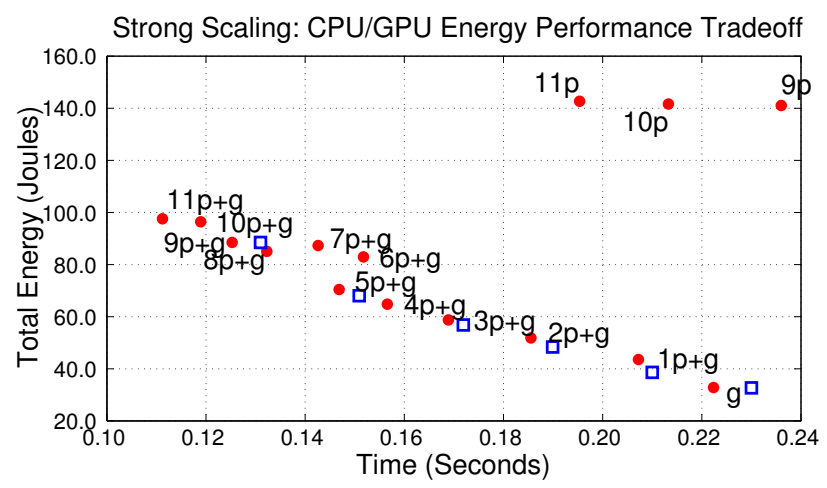

Fig. 14. Energy-performance tradeoff. 'p' stands for core and 'g' for GPU. The results are for matrix size $16 \times 16 \times 16$ and number of spectral elements is 10,000 . The most energy efficient implementation is that on a GPU while the implementation with the least runtime is one on 11 cores and GPU. The CPUonly implementations consume large amount of energy. Red circles represent performance optimal load balancing, whereas blue squares represent energy optimal load balancing.

improves proportionally as more cores are used in the computation, but power consumption also increases propor- 
tionally as the number of cores is increased. For CPU+GPU configurations, adding more CPU cores improves performance only slightly but power consumption increases rapidly. Performance of the CPU+GPU configurations is significantly better than the CPU-only configurations (from $x$-axis), while power consumption is comparable (from $y$-axis of these two figures).

Figure 12(d) shows the performance per Watt in terms of GFLOPS/Watt which is represented in log scale on the y-axis of the graph. Performance per Watt improves by an order of magnitude when a GPU is used, and for all matrix sizes the highest performance per Watt is obtained when the GPU is used by itself.

3) Energy: Figure 13 gives the energy consumed by the CPU-only and the CPU+GPU configurations. The energy consumption for the CPU-only configurations is nearly identical as cores are increased because improved performance is balanced by an increase in power as more CPU cores are added. For CPU+GPU configurations however, adding more CPU cores causes a steady rise in energy consumption since performance does not improve in the same proportion as power consumption.

4) Performance versus Energy Tradeoff: Figure 14 gives the performance and energy consumption of the various hybrid configurations we experimented with. Matrix size was set to $16 \times 16 \times 16$ and the total number of elements was set to 10000 for every case. The number of elements processed by the CPU cores or by the GPU device is set such that performance for the system is optimal. Though the GPU-only configuration consumed the least energy, we can improve performance by adding more CPU cores to the GPU to share the load. The pareto front of this multiobjective optimization problem is shown in blue in the figure.

The energy performance tradeoff can also be cast as an energy optimization problem under timing constraints which may be formulated as follows:

$$
\begin{array}{ll}
\underset{Y_{C P U}, Y_{G P U}}{\operatorname{minimize}} & E_{p C P U}+E_{g G P U} \\
\text { subject to } & T_{p C P U} \leq T_{1} \\
& T_{g G P U} \leq T_{1} \\
& Y_{C P U}+Y_{G P U}=Y_{1} \\
& g \leq G_{1} \\
& p \leq P_{1}
\end{array}
$$

where $p$ and $g$ are the number of CPU cores and GPU devices respectively, $T_{1}$ is the target runtime, $Y_{1}$ is the total number of elements, $G_{1}$ is the number of devices accessible from the node, whereas $P_{1}$ is the total number of cores on the node. Note that we do not constrain the various processing units to finish processing at the same time as we did to obtain optimal performance in Section IV-D3. So, the CPU cores in this case for example, could finish earlier than any of the GPU devices.

$E_{p C P U}$ and $E_{g G P U}$ are the energy consumed by $p \mathrm{CPU}$ cores and $g$ GPU devices and are modeled as follows since power consumption is directly proportional to the number of cores or devices used:

$$
\begin{aligned}
& E_{p C P U}=p \times P_{C P U} \times T_{p C P U} \\
& E_{g G P U}=g \times P_{G P U} \times T_{g G P U}
\end{aligned}
$$

which is interesting because the computation time taken by $p$ cores, $T_{p C P U}$, is $1 / p$ times the time taken by a single CPU, and similarly for the $g$ devices from equations 9 and 15. Therefore, $p$ and $g$ factor out from the energy expressions, and the optimization goal may be restated as:

$$
\underset{Y_{C P U}, Y_{G P U}}{\operatorname{minimize}} P_{C P U} \times T_{C P U}+P_{G P U} \times T_{G P U}
$$

which is effectively minimizing energy consumption on a single core and a single device. Therefore, by solving the above optimization problem we are able to make a recommendation of how many elements must be processed by the GPU and the CPU core to keep the energy consumption minimum and from the timing constraints we make a recommendation of how many processing units of each kind $(p, g)$ may be used to process the elements thereby meeting the timing constraints. It is possible to have multiple $(p, g)$ that satisfy the timing constraint in which case we should choose the one that consumes the least power.

For example, if we set the total time, $T_{1}$, to 0.15 seconds for processing $Y_{1}=10,000$ elements, where the matrices are $16 \times 16 \times 16(N=16)$, then substituting for $N$ in the models for GPU and CPU timing, power and energy (derived in Section IV-D), we rewrite the optimization problem as:

$$
\begin{array}{cl}
\underset{Y_{C P U}, Y_{G P U}}{\operatorname{minimize}} & 2.62 \times 10^{-3} \times Y_{G P U}+ \\
& 13.26 \times 10^{-3} \times Y_{C P U} \\
\text { subject to } & Y_{G P U} / g \leq 6995 \\
& Y_{C P U} / p \leq 746 \\
& Y_{C P U}+Y_{G P U}=10000 \\
& g \leq 1 \\
& p \leq 12
\end{array}
$$

Since the coefficient of $Y_{G P U}$ is smaller, therefore $Y_{G P U}$ has to be as large as possible, which is, $Y_{G P U}^{*}=6995$. And $Y_{C P U}^{*}=10000-6995=3005 . p$ is any integer less than or equal to 12 which satisfies the condition $3005 / p \leq 746$. In particular, we could set $p=5$. Of course, from a power point of view the fewer the $p$ and $g$, the lower the power consumption. Theoretically, the minimum energy is $2.62 \times 10^{-3} \times 6995+13.26 \times 10^{-3} \times 3005=58.17 \mathrm{~J}$. Experimentally, the runtime and energy consumption of this configuration ( $p=5, g=1$ ) are $149.98 \mathrm{~ms}$ and $64.08 \mathrm{~J}$ respectively. The energy consumed by the most performance efficient configuration is $70.41 \mathrm{~J}$ with a runtime of $146.84 \mathrm{~ms}$ from Figure 14. Thus, we got $9 \%$ more energy efficiency by sacrificing a $2 \%$ on performance.

Hence given a deadline, the least energy consumption is achieved when the GPU processes as much load as can be processed within the deadline with the remaining load being processed by the CPU cores. The table in Figure 15 


\begin{tabular}{|c|c|c|c|c|c|c|c|c|}
\hline \multirow{2}{*}{$\begin{array}{l}\text { Deadline } \\
\text { (sec) }\end{array}$} & \multicolumn{4}{|c|}{ Energy Optimal Results } & \multicolumn{4}{|c|}{ Performance Optimal Results } \\
\hline & Runtime (s) & $\begin{array}{l}\text { Peak } \\
\text { Power(W) }\end{array}$ & Energy $(\mathrm{J})$ & $\begin{array}{l}\text { GPU load (\% ele- } \\
\text { ments) }\end{array}$ & Runtime (s) & $\begin{array}{l}\text { Peak } \\
\text { Power(W) }\end{array}$ & Energy $(\mathrm{J})$ & $\begin{array}{l}\text { GPU load (\% ele- } \\
\text { ments) }\end{array}$ \\
\hline 0.230 & 0.2223 & 147 & 32.68 & 100 & 0.2223 & 147 & 32.68 & 100 \\
\hline 0.210 & 0.210 & 213 & 38.6 & 94.46 & 0.2072 & 213 & 43.5 & 91.25 \\
\hline 0.190 & 0.1899 & 279 & 48.37 & 85.47 & 0.1855 & 279 & 51.8 & 82.83 \\
\hline 0.172 & 0.1719 & 345 & 56.8 & 85.47 & 0.1689 & 345 & 58.72 & 76.58 \\
\hline 0.151 & 0.1509 & 411 & 68.05 & 67.27 & 0.1468 & 477 & 70.1 & 70.72 \\
\hline 0.137 & 0.137 & 675 & 88.5 & 51.14 & 0.132 & 675 & 89.31 & 49.22 \\
\hline 0.126 & 0.1259 & 741 & 93.22 & 44.56 & 0.1189 & 807 & 95.17 & 43.75 \\
\hline
\end{tabular}

Fig. 15. Energy optimal and performance optimal results for some given deadlines. The energy optimal use cases consume lower power for deadlines 0.151 seconds and 0.126 seconds, because these use one less CPU core in the computation.

gives some deadlines and the runtime, power, energy and load processed statistics for energy optimal and performance optimal implementations with $N=16$ and $Y=10000$.

5) Ratio of CPU to GPU Loads: Figure 16 gives the ratio of the number of spectral elements processed by GPU and CPU cores for optimal performance. As the number of CPU cores is increased, more load is processed by them. Compared to the other matrix sizes, the GPU is more efficient in processing the matrix size $16 \times 16 \times 16$. Hence, for this matrix size, load is balanced when more spectral elements are processed by the GPU than by the CPU cores. The workload ratio between GPU and a single CPU core for matrix size $16 \times 16 \times 16$ is 9:1 .

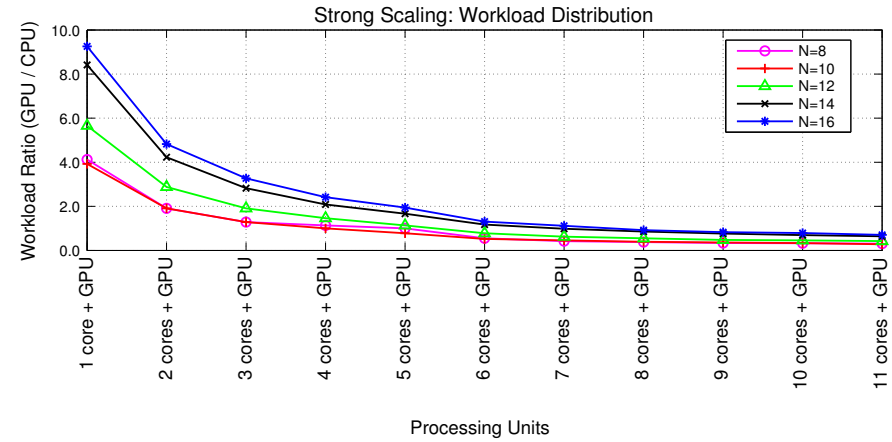

Fig. 16. Ratio of the number of spectral elements processed by the GPU versus CPU cores for optimal overall performance. For the matrix size $16 \times$ $16 \times 16$, GPU processes about 9 times more spectral elements compared to a single core to balance the load.

Figure 17 gives the ratio of the number of elements processed by the GPU versus those processed by the CPU if we ignore communication between CPU and GPU and focus only on kernel performance. Matrices of size $16 \times 16 \times 16$ are processed 100 times and 9 times faster on a GPU than on a single core and eleven cores of CPU. respectivelv.

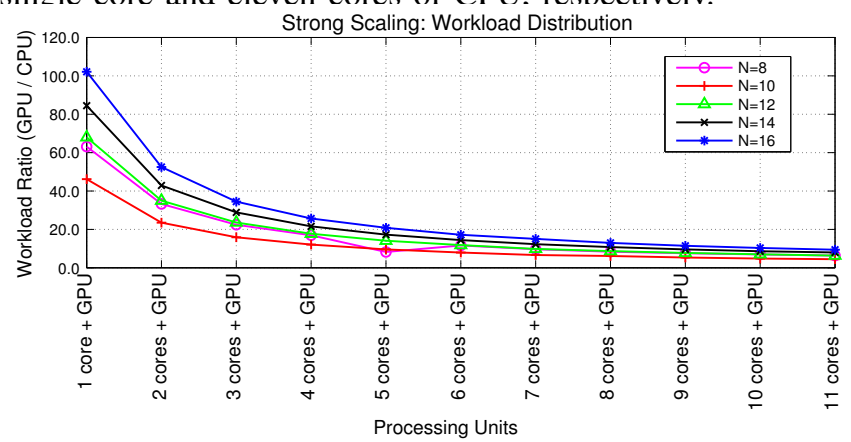

Fig. 17. Ratio of the number of spectral elements processed by the GPU versus CPU cores for optimal kernel performance. For the matrix size $16 \times$ $16 \times 16$, GPU processes about 100 times more spectral elements to balance the load compared to a single CPU core and about 9 times more load than 11 CPU cores.

\begin{tabular}{|l|l|l|l|l|}
\hline Matrix size & Processing Unit & Runtime(s) & Power(W) & Energy(J) \\
\hline $8 \times 8 \times 8$ & 1 core & 0.1095 & 68.65 & 7.63 \\
$8 \times 8 \times 8$ & 11 cores & 0.01015 & 755.15 & 7.66 \\
$8 \times 8 \times 8$ & GPU & 0.0272 & 148 & 4.03 \\
$8 \times 8 \times 8$ & 11 cores + GPU & 0.007 & 903 & 6.32 \\
\hline $10 \times 10 \times 10$ & 1 core & 0.2188 & 69.75 & 14.12 \\
$10 \times 10 \times 10$ & 11 cores & 0.0192 & 731.78 & 14.01 \\
$10 \times 10 \times 10$ & GPU & 0.0540 & 144 & 8.31 \\
$10 \times 10 \times 10$ & 11 cores + GPU & 0.0153 & 885 & 13.39 \\
\hline $12 \times 12 \times 12$ & 1 core & 0.5263 & 67.45 & 35.50 \\
$12 \times 12 \times 12$ & 11 cores & 0.0472 & 741.97 & 35.03 \\
$12 \times 12 \times 12$ & GPU & 0.0929 & 155 & 14.40 \\
$12 \times 12 \times 12$ & 11 cores + GPU & 0.0339 & 896 & 30.37 \\
\hline $14 \times 14 \times 14$ & 1 core & 1.25 & 67.37 & 84.21 \\
$14 \times 14 \times 14$ & 11 cores & 0.1144 & 741.07 & 84.83 \\
$14 \times 14 \times 14$ & GPU & 0.1509 & 153 & 22.92 \\
$14 \times 14 \times 14$ & 11 cores + GPU & 0.0704 & 894 & 62.50 \\
\hline $16 \times 16 \times 16$ & 1 core & 2.1216 & 66.39 & 140.85 \\
$16 \times 16 \times 16$ & 11 cores & 0.1953 & 730.32 & 142.66 \\
$16 \times 16 \times 16$ & GPU & 0.2224 & 147 & 32.69 \\
$16 \times 16 \times 16$ & 11 cores + GPU & 0.1112 & 877 & 97.52 \\
\hline
\end{tabular}

Fig. 18. Compute time, power and energy for different combination of processing units. For 11 cores + GPU, runtime drops though power rises, still energy is still significantly lower compared to that of 1 or 11 cores.

6) Overall comparison: The table in Figure 18 presents the performance, power and energy consumption of a CPU core, a GPU and a CPU+GPU configuration using $11 \mathrm{CPU}$ cores. For all the matrix sizes, the CPU+GPU configurations improves performance by up to $94 \%$ with respect to CPU and by more than $50 \%$ with respect to GPU. Though there is an increase in power upon adding the CPU cores to the GPU, overall energy consumption improved by more than $10 \%$ with respect to CPU. Overall, the GPU only configuration has the least energy consumption, which is more than $46 \%$ lower than the CPU-only energy consumption and 36\% lower than the CPU+GPU energy consumption.

\section{RELATED WORK}

GPUs have been used previously in the implementation of flow computation problems. Jacobsen et. al in [20] analyzed incompressible flow computations involving stencil computing. Cecka et. al in [21] proposed implementation of Galerkin methods on GPUs. Bolz et. al presented solutions to sparse linear system of equations resulting from finite element discretization in [23]. L. Wang et. al in [25] studied the limitations of scaling on CPU-GPU clusters. Dong et al. in [9] proposes the redesigning of a compressible hydrodynamics with high order finite elements on CPU+GPU architecture. Song et. al in [6] proposes tiling algorithms for QR and Cholesky factorizations on CPU+GPU architectures. Our application using spectral element method requires different strategies for optimization that the existing methods. Garcia et. al in 
[4] gives optimization and modeling techniques for matrix multiplication for large matrices on hybrid platforms.

Some of the other applications implemented on heterogeneous systems are comparison of biological sequences. Theoretical analysis of performance, energy and temperature optimization through scheduling and load balancing were studied in [11], [12] and [13]. Khan et al. in [11] applied cooperative game theory to the problem of allocation of tasks to computational grids. Ahmed et al. in [12] use game theory concepts for scheduling tasks whereas Hafiz et al. in [13] present an evolutionary algorithm for scheduling tasks based on multi-objective optimization of performance, energy and temperature.

\section{CONCLUSIONS}

We have benchmarked power performance characteristics of the derivative computation kernel which is one of the most compute intensive kernel of spectral solvers, on HMPs. Our optimization techniques on Nvidia Tesla K20c GPU leveraging the data reuse characteristics of spectral solvers got us performance of 180 gigaFLOPS, which is $39 \%$ better than what we get with the best existing implementation on GPU for small matrix-matrix multiplications.

We developed empirical models of performance, power and energy consumption of the derivative computation kernel on HMPs, which helped us devise our load balancing strategies. We showed that depending upon whether we want performance optimal or energy optimal implementation, we must use different load balancing strategy on HMP.

Thus, with the computational nodes powered by GPUs on exascale supercomputers, applications such as CMT-nek would benefit from a revised computational load allocation per node. In the future, we will integrate our code with CMT-nek and also experiment with multiple GPUs.

\section{ACKNOWLEDGEMENTS}

We would like to thank the members of the Center for Compressible Multiphase Turbulence at the University of Florida for their valuable feedback throughout the work. This work was funded by the U.S. Department of Energy, National Nuclear Security Administration, Advanced Simulation and Computing Program, as a Cooperative Agreement under the Predictive Science Academic Alliance Program, Contract No. DOE-NA0002378.

\section{REFERENCES}

[1] The Top500 List, June 2014 [Online]. Available: http://www.top500.org/lists/2014/06/

[2] DOE, Architectures and technology for extreme scale computing, December 2009

[3] T. Banerjee, S. Ranka, Genetic Algorithm based Autotuning Approach for Performance and Energy Optimization, IEEE International Green and Sustainable Computing, 2015.

[4] L-P. Garcia, J. Cuenca, D. Gimenez, On optimization techniques for the matrix multiplication on hybrid CPU+GPU platforms, Annals of Multicore and GPU Programming, 1, 1, 2014.
[5] R. C. Whaley, A. Petitet, J. Dongarra, Automated Empirical Optimizations of Software and the ATLAS project, Parallel Computing, 27, 1-2, pp. 3-35, 2001.

[6] F. Song, S. Tomov, J. Dongarra, Enabling and scaling matrix computations on heterogeneous multi-core and multi-GPU systems, ACM/IEEE Conference on Supercomputing 2012.

[7] R. Vuduc, J. W. Demmel, K. A. Yelick, OSKI: A library of automatically tuned sparse matrix kernels, SciDAC, June, 2005.

[8] C. Jhurani, P. Mullowney, A GEMM interface and implementation on NVIDIA GPUs for multiple small matrices, Journal of Parallel and Distributed Computing Spetember 2014.

[9] T. Dong, V. Dobrev, T. Kolev, R. Rieben, S. Tomov, J. Dongarra, A Step Towards Energy Efficient Computing: Redesigning a Hydrodynamic applicationon CPU-GPU, IPDPS 2014.

[10] J. H. Laros, III, P. Pokorny, and D. DeBonis, PowerInsight - A Commodity Power Measurement Capability, PMP, 2013.

[11] S.U. Khan, and I Ahmad, A cooperative game theoretical technique for joint optimization of energy consumption and response time in computational grids, Parallel and Distributed Systems, IEEE Transactions, 20 (3), pp 346-360

[12] I. Ahmad, S. Ranka, and S.U. Khan, Using game theory for scheduling tasks on multi-core processors for simultaneous optimization of performance and energy, IEEE International Symposium on Parallel and Distributed Processing, 2008

[13] H. F. Sheikh, I. Ahmad, and D. Fan, An Evolutionary Technique for Performance-Energy-Temperature Optimized Scheduling of Parallel Tasks on Multi-Core Processors, IEEE Transactions on Parallel and Distributed Systems (TPDS), 27(3), 2016, pp 668 681

[14] J. Lang, G. Runger, High-Resolution Power Profiling of GPU Functions Using Low-Resolution Measurement, Euro-Par 2013.

[15] P. Du, R. Weber, P. Luszczek, S. Tomov, G. Peterson, J. Dongarra, From CUDA to OpenCL: Towards a Performanceportable Solution for Multi-platform GPU Programming, Journal of Parallel Computing 38, 8, pp, 391-407, August, 2012.

[16] P. Fischer, J. Lottes, Hybrid Schwarz-Multigrid Methods for the Spectral Element Method: Extensions to Navier-Stokes, Domain Decomposition Methods in Science and Engineering, vol 40, pp 35-49, 2005.

[17] NVIDIA CUDA Basic Linear Algebra Subroutines (cuBLAS) library, Feb 21, 2013 [Online]. Available: https://developer.nvidia.com/cublas.

[18] M. T. Schmitz, B. M. Al-Hashimi, Considering Power Variations of DVS Processing Elements for Energy Minimisation in Distributed Systems, International Symposium on System Synthesis, pp.250-255, Oct 2001.

[19] S. Tomov, R. Nath, P. Du and J. Dongarra, MAGMA User's Guide. Technical report, ICL, UTK, 2011.

[20] D. A. Jacobsen, J. C.Thibault, I. Senocak, An MPI-CUDA Implementation for Massively Parallel Incompressible Flow Computations on Multi-GPU Clusters, 48th AIAA Aerospace Sciences Meeting and Exhibit, 2010.

[21] C. Cecka, A. Lew, E. Darve, Assembly of finite element methods on graphics processors, Numerical Methods in Engineering, Vol 85, No. 5, February 2011.

[22] PCI-SIG, PCI Express Base Specification Revision 3.0, 2010

[23] J. Bolz, I. Farmer, E. Grinspun, P. Schroder, Sparse Matrix Solvers on the GPU: Conjugate Gradients and Multigrid, $A C M$ Transactions on Graphics, 2003.

[24] N. Godel, N. Nunn, T. Warburton, M. Clemens, Scalability of Higher-Order Discontinuous Galerkin FEM Computations for Solving Electromagnetic Wave Propagation Problems on GPU Clusters, Magnetics, IEEE Transactions, Aug 2010.

[25] L. Wang, W. Jia, X. Chi, Y. Wu, W. Gao, L-W. Wang, Large Scale Plane Wave Pseudopotential Density Functional Theory Calculations on GPU Clusters, SC, 2011. 
[26] B. van Werkhoven, J. Maassen, F.J. Seinstra, H.E. Bal, Performance models for CPU-GPU data transfers, CCGrid, 2014.

[27] R. Bleuse, T. Gautier, J.V.F. Lima, G. Mounie, D. Trystram, Scheduling Data Flow Program in XKaapi: A New Affinity Based Algorithm for Heterogeneous Architectures, Euro-Par, 2014.

[28] Khronos Group, OpenCL, www.khronos.org/opencl/, 2013

[29] Nvidia, NVML Reference Manual, http://developer.download.nvidia.com/assets/cuda/files/ CUDADownloads/NVML/nvml.pdf, Version 3.295.45, April 20, 2012. 